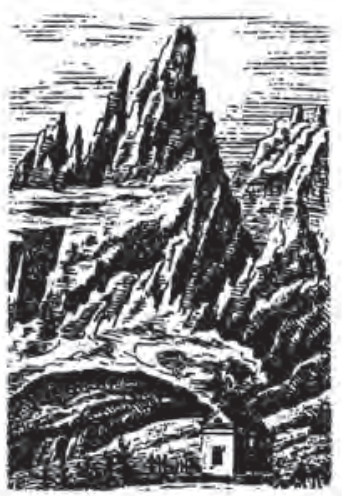

\title{
Anja Burghardt
}

Ludwig-Maximilians-Universität, München

anja.burghardt@Imu.de

https://doi.org/10.19195/2084-4107.13.13

\section{Gebirgs-Wege: Alpen- und Tatra- Impressionen in der polnischen Literatur des 19. Jahrhunderts}

Słowa-klucze: góry, krajobraz, Tatry, Alpy

Keywords: mountains, landscape, Tatras, Alps

\section{Mountain pathways: Alpine and Tatra impressions in nineteenth-century literature}

\section{Summary}

The author of the article analyses travelogues associated with the Alps and the Tatras by Polish nineteenth-century writers. She seeks to demonstrate the extent to which both the Alps and the Tatras - often appearing in Polish literature as a clear motif or theme - were idealised. The main source material analysed by the author comprises works by Juliusz Słowacki, Seweryn Goszczyński, Adam Asnyk, Maria Konopnicka, Bolesław Prus and Jan Kasprowicz. In her analysis the author stresses the interpretation of aquatic motifs associated with the mountain landscape, particularly lakes, which often seem to link the mountains to the sky. A comparison of the various ways of presenting the mountain landscape in works by Polish authors originating in various literary periods has revealed a range of literary idealisation processes. 
Als „Symbol der ewigen Dauer“ („wieczny symbol trwań“ bzw. „symbol wiecznych trwań") lässt Jan Kasprowicz die Jungfrau gleich zweimal in seinem Band Z Alp (Aus den Alpen) vor den Augen der Lesenden erstehen. Und die Gedichte in dem Zyklus Jungfrau sind nur drei von vielen, in denen die Natur über Zuschreibungen des Dauerhaften, Ewigwährenden und letztlich Göttlichen — in diesem Gedicht v.a. im Hinblick auf eine zeitliche Dimension - idealisiert wird. Kasprowicz ist in der Literatur freilich nicht der Einzige, der in den Bergen Symbole für das Himmlische und Unvergängliche findet. Die vielfältigen Gebirge-Texte unterscheiden sich aber darin, was das Bemerkenswerte der Berge ausmacht oder gar ihre Idealisierung gründet. Ausgehend von Francesco Petrarcas Die Besteigung des Mont Ventoux mag man den Blick von den Bergspitzen herab auf das scheinbar endlose Panorama einer Gebirgslandschaft und die Täler bzw. die Ebene, der neue Erkenntnisse eröffnet, als ein zentrales Moment in der Darstellung der Berge erwarten. Ein anderer Aspekt, der sich verschiedentlich in der Literatur findet, sind Abgeschiedenheit und Stille. So bilden entlegene Gebirgstäler in Miguel de Cervantes' Don Quijote einen Rückzugsort für Personen, die aus der Normalität herausgefallen sind: ${ }^{1}$ romantische Gedichte gründen das Gebirge-Ideal nicht selten auf einen Rückzug in Einsamkeit und Ruhe, wie beispielsweise Joseph von Eichendorffs Abschied: Nach einer Adressierung der „Täler in der Weite“ und der „Höhen“ rücken der Wald und seine Stille, die dem Lärm der Städte gegenübergestellt werden, ins Zentrum der Betrachtung. ${ }^{2}$ Denkt man an Mickiewicz' Sonety krymskie (1826, Krimsonette), so dominiert das Moment des Erhabenen der Berge, das einerseits auf der Schönheit wie auch auf der Höhe der Krimberge gründet, insbesondere des Czatyrdah, der in den Wolken verschwindet (vgl. das dreizehnte Sonett) und wo - wie der Mirza sagt — nichts als Sterne über einem stehen (fünftes Sonett), anderseits mit der Steilheit und der Tiefe von Schluchten verbunden ist (fünfzehntes Sonett).

In den folgenden Lektüren verschiedener Gebirgstexte der polnischen Literatur des 19. Jh. liegt das Augenmerk aber nicht so sehr auf solch einem Rückzug oder auf den Ausblicken, die eher beiläufig aufscheinen. Im Zentrum des Interesses stehen vielmehr zum einen Farben und Lichtverhältnisse, zum anderen das Wasser, oft Gebirgsbäche und Wasserfälle, insbesondere aber Seen. Wie aufgezeigt wird, erfährt über deren Idealisierung auch die Berglandschaft insgesamt eine solche. Dabei wird auch der Frage nachgegangen, welche sprachlichen Mittel für die Markierung des Ideals genutzt werden und wie sich diese gegebenenfalls über die Epochen hinweg wandeln. In chronologischer Reihenfolge werden die folgenden Texte betrachtet: Juliusz Słowacki: W Szwajcarii (1836, In der Schweiz), Seweryn Goszczyński: Dziennik podróży do Tatrów (1834/1853, Tagebuch meiner Tatrareise), Adam Asnyk: W Tatrach (1880, In der Tatra), Maria Konopnicka: Wrażenia z podróży (1883, Rückkehr von der Reise), Bolesław Prus: Wędrówka po ziemi i niebie (1887, Himmel und

${ }^{1}$ Vgl. dafür im ersten Teil die Episoden in der Sierra Morena, in die sich Cardenio und dann der „Ritter von der traurigen Gestalt“ zurückziehen; auch Dorotea flüchtet dorthin, nachdem sie von ihrem Geliebten verlassen wurde.

2 Eichendorff 2006, S. 346 f. 
Erde: ein Streifzug) sowie Jan Kasprowicz: Na Jeziore czterych kantonów (1895, Am Vierwaldstättersee). In der Auswahl wurde darauf geachtet, dass für die beiden in der polnischen Literatur sehr präsenten Gebirge, Alpen und Tatra, Texte zumindest aus zwei literarischen Epochen gegeben sind. In diesem vergleichenden Blick auf die Idealisierung der Berge spiegeln sich freilich wohl bekannte ästhetische Vorstellungen der unterschiedlichen Epochen wider. Die Konkretheit der Textanalysen wird hoffentlich verhindern, dass das Folgende wie eine Re-Iterieration dieser Entwicklungen erscheint. Vorweggenommen sei allerdings, dass sich im Zusammenhang mit der Frage nach der Idealisierung für die Prosa des Positivismus (nicht so sehr für die Lyrik) mit ihrem Streben nach mimetischer Treue die Frage stellt, ob hier eigentlich eine Idealisierung des Gegenstands überhaupt denkbar ist.

Wie mit den „Gebirgs-Wegen“ im Titel angedeutet, handelt es sich bei den meisten der hier vorgestellten Texte um Reiseliteratur, also um Texte, die auf tatsächliche Reisen der Autorinnen und Autoren zurückgehen und in denen sie — sehr unterschiedlich in der poetischen Überformung — diese Reise darstellen. Eine Ausnahme bietet hier Słowackis Schweiz-Poem, das ein Liebesgedicht darstellt. ${ }^{3}$ Auf im weitesten Sinne ethnologische Annäherungen an die Kultur der Góralen in der Tatra wird nicht näher eingegangen.

\section{Juliusz Słowacki: Berge als Spiegel der Liebe}

Słowackis Poem W Szwajcarii lässt sich als Beispiel für eine romantische Idealisierung der Berge lesen. ${ }^{4}$ Der Sprecher erzählt von seiner großen Liebe zu einer (natürlich wunderschönen) jungen Frau, deren Tod gleich zu Beginn des Poems genannt ist. Die ursprünglich gegenseitige und glückserfüllte Liebe scheint letztlich durch eine körperliche Annäherung der Liebenden getrübt, wobei diese sinnliche Erfüllung latent als (Mit-)Grund für den Tod der Geliebten erscheint. ${ }^{5}$ Verschiedentlich fließen in dem Text die Geliebte und verschiedene Naturerscheinungen ineinander. Die Schönheit, Reinheit und Unschuld der Angebeteten und das Überwältigende der Natur wirken wechselseitig aufeinander zurück, so dass

3 Słowacki lebte von 1832-1836 in der Schweiz, in Genf; er begab sich 1834 und 1835 auf Reisen und Wanderungen in die Berge. Dem Poem liegt also durchaus eine Kenntnis der Landschaft zugrunde. Maciejewski (1974) legt dar, wie sich Słowackis Stilisierung der Landschaft, die sich an sentimentalistischen Landschaftsdarstellungen des illustrierten Reise-Albums orientiert, in diesem Poem zu den Realia seiner Reise verhält, 96-98.

${ }^{4}$ Zum Bild der Alpen in der polnischen Romantik allgemein unter Einbettung in die europäische „Alpenliteratur“ (111) vgl. Kolbuszewski 1993; ausführlich diskutiert Słowackis Alpendarstellung Makowski 1976; zu biographischen Hintergründen vgl. Sawrymowicz/Makowski 1960; in der hier verwendeten neuen Ausgabe der Poeme wird im Kommentar ebenfalls auf Realien und Hintergründe verwiesen.

5 Für eine detaillierte Strukturanalyse, aufgrund derer die europäische Liebespoesie seit Petrarca als Kontext für das Poem erscheint, vgl. Bischof Hayoz 2016, Teil C, Kapitel 2, 259-316. 
letztlich beides überhöht wird. ${ }^{6}$ Von Anfang an spielen Bilder des Fließens, der Klarheit und der Helligkeit eine wichtige Rolle; die Bergwelt ist geprägt von Farbenreichtum, wobei die Farbe von Rosen sowie Silber und Gold besonders präsent sind. Die wiederholte Nennung des Regenbogens verleiht der Szenerie eine entsprechende Farbigkeit, Leuchtkraft und Transparenz, deren Dichte und beinahe Ausschließlichkeit zur Idealisierung führt. ${ }^{7}$ Hier ein kurzes Beispiel aus dem zweiten Abschnitt des Poems (also zu Beginn der eigentlichen Geschichte), als der Sprecher seiner Geliebten erstmals begegnet:

W szwajcarskich górach jest jedna kaskada, Gdzie Aar wody błękitnymi spada.

$[\ldots]$

Tam ją ujrzałem!

[...]

Tak jasną była od promieni słońca!

Tak pełna w sobie anielskiego świtu!

Tak rozwidniona zrennicą z błękitu! -

In den Schweizer Bergen gibt es einen Wasserfall, in dem das Aar-Wasser himmelblau herabstürzt.

[...]

Dort erblickte ich sie!

[...]

So hell war sie von der Sonne Leuchten!

So erfüllt von Engelslicht!

So erhellt das Auge von Azur!

Auch die Begegnung und Liebeserklärung der Geliebten in der Eisgrotte sind ein einziges Spiel von Licht, Weiße und Helligkeit. Beispielsweise beginnt der 7. Abschnitt mit einem Bild der Eisgrotte, die ganz von Licht geprägt ist:

Raz mię ów anioł zaprowadził złoty

Przesz jasne łąki do lodowej groty.

Tam ją obielił dzień alabastrowy;

I mróz na czole mej jasnej królowej

Perłami okrył wszystkie polne róże.

Einmal führte mich jener goldene Engel

Über helle Wiesen zu einer Eisgrotte.

Dort machte sie der Alabastertag weiß;

${ }^{6}$ Makowski (1976) liest die Antonimien (Leben bzw. Liebe vs. Tod, Idylle vs. Tragödie, Bewegtheit und Wandel vs. Bewegungslosigkeit, Freiheit vs. Begrenztheit) als Ausdruck „,des komplizierten menschlichen Inneren“ (,wyrażającą skomplikowane ludzkie wnętrze“, 55).

7 Freilich steht dem im weiteren Verlauf des Poems eine Düsternis zur Seite. Aus der vorangegangenen Idealisierung ergibt sich erst deren Ausmaß. 


\section{Und der Frost auf der Stirn meiner hellen Königin}

Umhüllte wie Perlen alle Feldrosen.

Die leuchtend-helle und lichtdurchdrungene Eiswelt bleibt bestimmend für die folgenden Strophen, ehe am Ende des Kapitels auch die Geliebte (wohl zum einzigen Mal in wörtlicher Rede), ihre Gefühle über die wärmende und die Leuchtkraft der Sonne beschreibt. Auch sie spricht von der „Helligkeit, geschaffen von der Sonne Leuchten“(„Ta jasność, słońca stworzona promieniem”), auf welche die beglückte Beschreibung der gemeinsamen Erlebnisse in den Bergen folgt. Wenn der Sprecher am Ende (im XX. Abschnitt) darüber reflektiert, dass er seine verstorbene Geliebte nicht zu zeichnen vermag, stehen abermals der Schnee und das abendliche Licht in den Alpen im Vordergrund.

\section{Seweryn Goszczyński: empfindungsreiche Landschafts-Detailliertheit}

Einer der wichtigsten Gebirgs-Texte der Romantik in Prosa dürfte Goszczyńskis Dziennik z podróży do Tatrów sein. ${ }^{8}$ Mit dem Ziel (so heißt es im Vorwort an die Lesenden), die Atmosphäre so einfühlungsreich und lebendig wie möglich zu vermitteln, ${ }^{9}$ erwähnt der Sprecher schon anfangs die beeindruckende Wildheit (dzikość) der Tatra. Er geht dann — sehr sachlich im Ton - auf die geographische Lage des Gebirges ein, ehe er von den zurückgelegten Wegen im Gebirge (immer wieder mit dem Ausblick auf Gipfel und Täler), von Ortschaften erzählt. Der Weg führte tatsächlich über einige Gipfel, die Beschreibung der Aussicht fällt aber gemeinhin recht kurz aus. Außerdem schreibt er Gedichte und Legenden der Góralen nieder. Als ein Höhepunkt der Reise erscheint der Weg zum Morskie Oko, dem Meerauge „in das Innere der Tatra“ (w głąb Tatrów). ${ }^{10}$ Die Idealisierung gilt aber weniger diesem See, von dessen Anblick der Sprecher fast enttäuscht ist, sondern vielmehr dem Wasserfall Siklawa und den Fünfseen („Pięciostawy“, wie sie Goszczyński bezeichnet). ${ }^{11}$ Diese beschreibt er ausführlich und in Superlativen — eben darin spiegelt sich die Idealisierung — und bekennt seine sprachlose Begeisterung:

8 Sierotwiński (2005) analysiert die Charakteristika des Werkes (vgl. zu Form und Komposition LV-LXIV, zur Thematik LXIV-LXVI), Goszczyńskis Reiseroute kommentiert er LXVI-LXXIII; vgl. hier auch für eine kommentierte Bibliographie; verwiesen sei noch auf die hier nicht angeführte Studie von Sosnowska (2000).

9 Vgl. dazu im Vorwort an den Leser („Dla czytających to dzieło“) $3 \mathrm{f}$.

10 Vgl. den Eintrag vom 13. August 1832, 176-190 im Kapitel „Wyprawa w głąb Tatrów“.

11 Vgl. ebda., S. 182-186. Das Meerauge erreichen die Wanderer vom Gipfel Świstowa Góra aus (cf. 186). 
...jakiej nic podobnego oczy moje dotąd nie widziały, a nawet wyobraźnia nigdy mi nie przedstawiła. ${ }^{12}$

...etwas Vergleichbares hatten meine Augen bislang noch nicht gesehen, und selbst meine Vorstellungskraft konnte sich so etwas nie ausmalen.

Ein Moment, das an den Wasserfällen beeindruckt, ist deren Fallhöhe. In dieser Hinsicht wird die Höhe auch in anderen Texten thematisiert, nämlich dass die Felswände beeindruckend und fast unbeschreiblich hoch sind. Farbe und Material des Steins scheinen dabei ein wichtiger Teil ihrer Eindrücklichkeit. Allerdings schwingt dabei nicht selten auch ein bedrückendes Moment mit, das in ihrer dunklen Färbung, ihrer Unbegehbarkeit und der Unmöglichkeit für Pflanzen (als Inbegriff des Lebendigen in der Bergeshöhe) sich in ihnen zu verwurzeln, gründet.

Nach diesem Blick auf zwei romantische Idealisierungen der Berge über die Schönheit (sei es der Geliebten, sei es der Landschaft), gelten die folgenden drei Beispiele dem Positivismus.

\section{Adam Asnyk: kontrastreiche Landschaftsbilder}

In seinem Zyklus $Z$ Tatr greift Adam Asnyk Topoi der Tatra-Texte auf, wie das Kościeliska-Tal oder den Gebirgssee Morskie Oko, die bereits im 19. Jahrhundert aus künstlerischen Annäherungen an dieses Gebirge kaum wegzudenken scheinen. ${ }^{13}$ Wie in der Literaturgeschichte immer wieder betont, zeichnet sich die polnische Lyrik des Positivismus in weiten Teilen durch ein Fortschreiben der romantischen Poetik aus. ${ }^{14}$ Das gilt sicher auch für Asnyks Tatra-Gedichte. ${ }^{15}$ Allerdings lässt sich eine größere Nähe zu Realien beobachten, teilweise verbunden mit der Suche danach, die äußeren Phänomene möglichst genau zu erfassen, anstelle einer Mitteilung des Empfindens, das sie auslösen (und das, wie erwähnt, Goszczyński wichtig ist; bei Słowacki ist dies fraglos tragend).

Das Eingangsgedicht ist eine Hommage an die „Ernährerin Mutter Erde“ („O matko ziemio, dobra karmicielko!“ beginnt es), in dem die Adressatin als Grundlage des menschlichen Lebens angerufen wird. Im wahrsten Sinne des Wortes erscheint sie als solche, wenn „dieser Boden“ (,ten grunt“, 7 Strophe), gewissermaßen als Verankerung der Menschen dient, so dass die Träume sie nicht in die Irre führen. Die Mutter Erde hat dem Menschen den Körper gegeben, wie sie auch seine Seele

12 Vgl. ebda., S. 183.

13 Kolbuszewski (1971) führt einschlägige Motive an, wie auch schon Pawlikowski (1934). Während Pawlikowski eine geographisch-thematische Herangehensweise wähle, stehe in seiner eigenen Untersuchung die Entwicklung der Tatramotive und -bilder im Mittelpunkt (7); aus der Malerei ist beispielsweise Jan Nepomucen Głowackis Gemälde „Morskie Oko w Tatrach“ (1837) bekannt.

14 Vgl. beispielsweise Markiewicz (2006).

15 Zu Asnyks Werk allgemein vgl. beispielsweise Mocarska-Tycowa (1990); vgl. auch Sokólska (2008). 
nährt (so der Beginn der 2. Strophe), und sie sorgt dafür, dass jeder sein Auskommen an einem für ihn geeigneten Platz findet. Wie bei Słowacki gibt es auch hier die „Strahlen der Sonnen-Helligkeit“(,,promienie jasności słonecznej“, 1. Strophe), aber während sie dort der doppelten Verherrlichung von Geliebter und Gebirge dienen, ist ihnen hier das Brot (als Metonymie für Nahrungsmittel) zu verdanken.

Die einzelnen Berggedichte sind sozusagen nach allen Regeln der Dichtkunst verfasste Bildsequenzen von variabler Länge und Gestalt: In wechselnden Metren ${ }^{16}$ verleihen syntaktische Parallelismen und Anaphern in Verbindung mit den markanten Sprachbildern etlichen Strophen ihre Eindrücklichkeit. Asnyk lässt in „Ranek w górach" (,Morgen in den Bergen“) die Hell-Dunkel-Kontraste und das Erwachen der Gebirgswelt sich entfalten. Als Textbeispiel sei eine Strophe aus dem fünfteiligen Zyklus über das Meerauge angeführt, dessen Sonette unterschiedliche Momente der Szenerie einfangen. Auch wenn Sonnenstrahlen vereinzelt in diese Felswelt einzudringen vermögen, herrschen insgesamt doch eher finstere Bilder vor. Im letzten Gedicht wird der Bergsee als „Poem“ adressiert, also als ein ausgefeiltes Kunstwerk der Natur. Widersprüche prägen dabei manches Gedicht; immer wieder ist von der riesigen Größe die Rede. In Hinblick auf eine Idealisierung scheint eine Totalität aussagekräftig, die sich zu Beginn des Zyklus zeigt:

Ponad płaszczami borów, ściśnięte zaporą

Ścian olbrzymich, co koło ze sobą się zwarły,

Ciemne wody rozlewa posępne jezioro

Odzwierciedlając w łonie głazów świat zamarły.

Über dem Mantel der Hochwälder, zusammengedrängt von einer Sperre Riesiger Wände, die sich im Kreis zusammenschlossen, vergießt der düstere See dunkle Wasser

die im Schoß die erstarrte Welt des Gesteins widerspiegeln.

Der See, der von Bergen, welche seine Gestalt bestimmen, umrahmt ist, fasst in sich diese Welt. Mit der Konzentration auf diese vielfältige Geschlossenheit, die im See zu einer Ganzheit verschmilzt, wird diese Reduktion mit einer extremen Ausweitung verbunden, die letztlich mit jedem der Sprachbilder im Gedicht weitere Facetten erhält. Die Vielfalt bleibt in der Konzentriertheit des Kreises beschlossen und hat so das Potenzial zum Nebeneinander von Gegensätzlichem.

\section{Maria Konopnicka: Berge als Ort von Sozialstudien}

Anders gelagert ist die Darstellung der Berge in Konopnickas Wrażenia z podróży, ein Prosatext, der einer Reportage nahekommt. Von Italien geht die Reise

16 Beispielsweise folgt einem 11-Silber mit einer Zäsur nach der 5. Silbe in der Einleitung ein vierhebiger Jambus; „Eine Nacht zu Füßen der Tatra-Spitze“ („Noc pod Wysoką“) weist als 11-Silber in seiner wiederkehrenden Rhythmik eine Nähe zu einem vierhebigen Daktylus auf. 
über Südtirol und Österreich; ${ }^{17}$ auf der Strecke zwischen Roveredo und Brixen sind die Wege und Pfade durchs Gebirge beschrieben. Landschaftsbeschreibungen sind meist kurze Sequenzen, die zwischen der Schilderung von Ortschaften, Unterkünften und immer wieder v. a. Menschen, denen die Reisende unterwegs begegnet, eingestreut. Das Reisen selbst ist ein weiteres Element dieser Reportage, und es erscheint durchaus als Abenteuer, so beispielsweise wenn eine ziemlich wackelige Brücke über die Etsch überquert werden muss. Die Naturschilderungen sind dabei reich an Attributen, auch ist von der Herrlichkeit (oder auch: Erhabenheit, Pracht: „majestat") der Schlucht bei Klausen die Rede, und sicher sind die Gebirgsschilderungen bildreicher als die Darstellungen der Ortschaften und ihrer Bewohner. Sie scheinen aber nicht idealisiert zu werden. Besonders deutlich wird das vielleicht mit dem Ortler, dem höchsten Berg dieses Alpenkamms, der mit seinem Gletschergipfel durchaus imposant ist (und in der bildenden Kunst des 19. Jahrhunderts mehrfach abgebildet wurde). ${ }^{18}$ Der Ortler wird genau mit einem Satz erwähnt und zwar ohne irgendwelche Schmuckelemente: Es ist eben diese Gebirgskette, an der der Weg entlang führt (vgl. 183). Weit mehr Aufmerksamkeit gilt den Arbeitern, die den Weg am Ufer der Etsch gebaut haben, sowie den wirtschaftlichen und gesellschaftlichen Verhältnissen in Tirol. Die Darstellung ist an dieser Stelle zwar eher allgemein gehalten, in dem Prosatext wird sie aber über die Porträts einzelner Personen immer wieder konkretisiert.

Meines Wissens verfasste Konopnicka im Rahmen ihrer Reise nach Österreich und Italien keine Gedichte über diese Berge — anders als bei den italienischen Orten, denen sie einen Zyklus von Sonetten, die Sonety wtoskie (Italiensonette) ${ }^{19}$, gewidmet hat. Insgesamt lässt sich die Darstellungsweise in den Italiensonetten, die eine von Licht durchflutete Landschaft entwerfen, in denen die Menschen eingangs als ,große Geister“ bezeichnet werden, durchaus als eine Idealisierung ansehen. Denn hier wird über Motive wie Licht und Meer, den Marmor, über Kunstwerke und Architektur ein in seiner Schönheit unübertreffliches Land entworfen. ${ }^{20}$ Vergleichbar mag die Rolle der Personen in beiden Texten sein: In den Italien gewidmeten Sonetten tragen die genannten Akteure immer wieder dazu

17 Ritz (2012) diskutiert - in Anknüpfung an Orzeszkowas „Ad Astra“ - Konopnickas „Miłosierdzie gminy“ als ein Beitrag zum Schweiz-Bild in der polnischen, aber auch in anderen Literaturen der zweiten Hälfte des 19. Jh.; zur Schweiz in der Lyrik verschiedener slavischer Sprachen vgl. Brang (1998), in seiner Einführung insbesondere den Abschnitt „Alpen, Kaukasus und Hohe Tatra“ (53-55). Für eine kritische Diskussion des Begriffs der „Berg-Literatur“ anhand einer Auseinandersetzung mit polnischen Anthologien zum Bergmotiv vgl. Dutka (2017).

18 Jochberger (2004) versammelt eine Reihe von Zeichnungen und Gemälden des Ortlers aus dem 19. Jh., beispielsweise von Wolfgang Pusch: „Der Suldenferner bedroht die Gampenhöfe in Sulden“ (1819) oder Heinrich Heinlein: „Hochgebirgslandschaft. Ortlergruppe“ (1845). Ob Konopnicka solche Kunstwerke kannte, ist mir nicht bekannt.

19 Nach der Veröffentlichung in verschiedenen Zeitschriften 1893-1989 wurde der Zyklus in dem Gedichtband Italia 1901 veröffentlicht, vgl. Zięba 1987; er hebt hervor, dass sich mit den Italiengedichten neue Themen und ein neuer Stil in der Lyrik der Dichterin abzeichnen.

${ }^{20}$ Besonders deutlich tritt die Schönheit im dritten Gedicht, Ranek w Wenecyi (Ein Morgen in Venedig), hervor. 
bei, dass die Städte und Orte, denen die Gedichte gelten, wenn nicht als etwas Ideales, so doch als Herausragendes erscheinen. Ähnlich lässt sich die Reise durch Südtirol lesen: Eine Idealisierung geschieht über die eine Landschaft gestaltenden Menschen.

In ihrem früher geschriebenen Zyklus W górach (1876, In den Bergen), der einem Aufenthalt in den Beskiden gewidmet ist, steht zwar die Gebirgslandschaft stärker im Vordergrund und in einzelnen Gedichten ist eine göttliche Schönheit benannt oder die Bilder für die Felslandschaft sind mit Emblemen von Reichtum und Herrschaft durchzogen. ${ }^{21}$ Dennoch scheint hier insofern größten Teils keine Idealisierung gegeben, als viele der Gedichte liedhafte Züge aufweisen; sie sind so gewissermaßen nicht feierlich genug, um als Ideal erscheinen zu können. Außerdem unterstreichen die vielen Hinweise auf Berggipfel, Ruinen oder auch Ortschaften die unternommene Reise, so dass die Intensität der einzelnen Szenerien, auf welcher eine Idealisierung immer wieder gründet, nicht so sehr in den Vordergrund tritt.

\section{Bolesław Prus: jenseits der Messbarkeit}

Bolesław Prus' Kartki z podróży sind meist Städten gewidmet; dezidierte Landschaftsdarstellungen gibt es eher am Rande. Die Reportage „Wędrówka po ziemi i niebie“ ist ebenfalls nicht wirklich eine Landschaftsdarstellung; ihr Gegenstand ist vielmehr die absolute Sonnenfinsternis (am 18. August 1887). Berge kommen hier zwar vor (er schildert frühere Ausflüge in Richtung Zakopane), die Eindrücke von ihnen sind aber durchaus zwiespältig. ${ }^{22}$

Vielsagend im Hinblick auf Fragen der Idealisierung scheint aber seine Darstellung der Sonnenfinsternis zu sein. ${ }^{23}$ Denn hier zeigt sich der Reporter, der sich gemeinsam mit einem Bekannten einer Gruppe von Wissenschaftlern angeschlossen hat, die Teil des (riesigen) Touristenstroms zu diesem Naturereignis sind, sehr beeindruckt von dem Naturschauspiel. Dass ein Ideal der Zeit die Messbarkeit ist, wird deutlich an den Mitreisenden des Reporters, die genau die Dauer der Sonnenfinsternis festhalten wollen (wie im Übrigen auch er es tun will). Zwar weichen die einzelnen Messergebnisse nur um Sekunden voneinander ab, allerdings durchaus signifikant, denn sie schwanken zwischen 87 und 114 Sekunden als Dauer für die vollkommene Verfinsterung der Sonne. Verstärkt wird die Fragwürdigkeit der Daten als Form von Welterkenntnis durch den Kommentar, dass der Reporter

${ }^{21}$ Allgemein zu Bergen in Konopnickas Euvre vgl. Jazowska-Gumulska (2002); Mazan (2011) stellt die Wahrnehmung der Realität und die Lebendigkeit der Schilderung in Konopnickas Dichtung in den Mittelpunkt seiner Untersuchung.

${ }^{22}$ Vgl. S. 93-95 für die Berge-Passagen.

23 Iwona Wojtaś (2017) diskutiert im Kontext einer Untersuchung des Motivs der Sterne bzw. des Sternenhimmels in Prus' Roman Lalka (Die Puppe) die Rolle der Astronomie in ausgewählten Texten. Allgemein zu Fragen von Wert und Bedeutung in allen drei hier in den Blick genommenen Epochen vgl. Igliński (2008). 
selbst zeitweise von den Geschehnissen um sich herum abgelenkt war und die Uhrzeit ganz vergaß (und die Vermutung liegt nahe, dass das auch für Leute mit einer größeren wissenschaftlichen Obsession als der seinen der Fall war). Dass Ungenauigkeit und Fragwürdigkeit der Zahlen gar nicht so wichtig sein könnten, scheint verschiedentlich in dieser Reportage auf. So heißt es an einer Stelle:

Jest niby ciemno, a jednak wszystko widać, jest mrok burzowy, lecz obłoki nie mają burzowego wyglądu. Oczy moje coś widzą, ale ja nie umiem tego określić. Brak mi nawet porównań. Tylko wielki żal, który rozpiera mi serce, może znaleźć porównanie. Gdyby konający widział własną twarz, jak mu stopniowo blednie, sinieje, wreszcie szarzeje, doznałby zapewne tego smutku, jaki się ma patrząc na stopniowe zamieranie światła.

Es ist als sei es dunkel, doch alles ist sichtbar, es ist dunkel wie bei einem Gewitter, doch die Wolken sehen nicht wie Gewitterwolken aus. Meine Augen sehen etwas, aber ich kann es nicht genau bestimmen. Selbst Vergleiche fehlen mir. Nur eine große Sehnsucht, die mir das Herz zu zerspringen droht, könnte als Vergleich dienen. Würde nur ein Sterbender das eigene Gesicht sehen, wie es bleich, blau und schließlich grau wird, könnte er genau diese Trauer erleben, wie das allmähliche Ersterben des Lichts in einem hervorruft.

In Naturerscheinungen gibt es also etwas, das über alltägliche Erfahrungen (man könnte hinzufügen: im städtischen Umkreis, denn Erklärungsversuche der Sonnenfinsternis zu Beginn dieser Reportage bedienen sich der Straßenbeleuchtung als Vergleichsmoment) hinausragt. Meines Erachtens lässt sich dieser Kommentar nicht eindeutig so interpretieren, dass die Natur oder das Naturerlebnis eine Idealisierung erfährt. Rein sprachlich verbleibt diese Passage in einer gewissen Nüchternheit, wenn sie sich auch von dem stellenweise ironischen Ton eines Gutteils der Reportage abhebt. Eindeutig ist aber die Kritik an der Erforschungsweise und - mehr noch — an dem Glauben, dass sich die menschliche Existenz mit diesen erfassen ließe (im Sinne eines Erkennens und Begreifens). Damit tritt v. a. ein bestimmter Wert (oder eine gewisse - von Werten getragene - Haltung) zutage: Es gilt, auch dem nicht begrifflich Fassbaren seine Gültigkeit zu lassen und einem - im weitesten Sinne emotionalen - Erleben des Menschen seinen Platz einzuräumen, da dieser fragloser und wichtiger Teil der menschlichen Existenz ist.

\section{Jan Kasprowicz: eine Sprachbilder-Feier der Berge}

Als Beispiel der Epoche Młoda Polska wird Jan Kasprowicz’ Alpen-Zyklus herangezogen. Der Band $Z$ wirchów i hal enthält zudem einen Tatra-Zyklus (Z Tatr); dieser ist in sich weit heterogener als die Gedichte, die während und nach der Schweiz-Reise entstanden sind. In den der Tatra gewidmeten Gedichten stehen 
Flora und Fauna neben Landschaftsbeschreibungen, einem Zyklus über den Wind (Wiatr halny) und folkloristischen Elementen, die teils mit ihrer formalen Gestalt, teils - wie beispielsweise in dem Gedicht Taniec zbójnicki (Räuberlied) — mit der Übernahme von Motiven aus der Volkskunst in Zakopane gegeben sind. Vor allem in der Abfolge der Gedichte ergibt sich daraus eher eine abwechslungsreiche Vielfalt als eine zunehmende Verdichtung der Bilder. Zweifellos spricht aus den Tatra-Gedichten eine Begeisterung für die Landschaft und Kultur; eine Idealisierung ist am ehesten in einzelnen Gedichten gegeben, beispielsweise im zweiten über den Adler, den „königlichen, stolzen Vogel“ (mit den Worten „Królewski, dumny ptak“ beginnt das Gedicht).

Der Band mit Gedichten über die Schweiz setzt mit einem Zyklus über den Vierwaldstätter See ein (Na jeziorze czterech kantonów). Hier gibt es eine ganze Reihe an Attributen und Charakterisierungen, mittels derer eine Sakralisierung geschieht oder - beispielsweise über Edelsteine - die für die Landschaftsdarstellungen gewählten Sprachbilder das Wertvolle unterstreichen und so zu ihrer Idealisierung führen. Beispielhaft sei eine Passage vom Beginn des ersten Gedichts zitiert, die — gar nicht unähnlich Asnyks „Meerauge“ — den See als Spiegel der Welt zelebriert, in dem alles zu einer Einheit findet:

Rozbłękitnioną płyniemy topielą...

Na jej zwierciadło snać się niebo zwali,

Ciężkie od blasków, co się po nim ścielą,

Lejąc szmaragdy wskroś stopionej stali.

Naokół góry, naodziane zielą

Marzących świerków, kąpią szczyty w fali;

Czasami śnieżne kopy się zabielą

Lub zaczernieją granity oddali.

Wir schwimmen durch die zerblaute Tiefe ...

Auf ihren Spiegel scheint der Himmel niederzustürzen, schwer von Glanz, der sich in ihm ausbreitet, da er Smaragde von geschmolzenem Stahl hinschüttet.

Ringsum Berge, bekleidet vom Kraut

Träumender Fichten, baden die Gipfel in den Wellen;

Hier und da schimmern verschneite Bergkuppen weiß

Oder Granit in der Ferne schwarz.

Das Gedicht fährt damit fort, dass der Wind nicht nur das Wasser in Bewegung setzt, sondern auch schläfrige Melodien und geheimnisvolle Botschaften (,wieści“) herbeiträgt. Die Szenerie sei insgesamt dazu angetan von Sorge und Leid der Menschen fortzuführen.

Kasprowicz' Gedichte sind von Neologismen geprägt, vom Rückgriff auf ein breites Spektrum an Wortfeldern und von einer bemerkenswerten Farbenvielfalt, 
die in einer innovativen sprachlichen Bildlichkeit kulminieren. ${ }^{24}$ Gewissermaßen geht in diesen Gedichten die Feier der Natur über in eine Feier der Ausdruckskraft der Sprache.

Mit der Vielzahl an Zitaten sollte angedeutet werden, wie unterschiedlich eine Idealisierung der Berge gestaltet ist. Nach der Konzentration auf die Rolle der Gewässer, insbesondere des Sees als Spiegel von Bergen und Welt, der eine verdichtete Eindrücklichkeit ermöglicht, bei der auch Widersprüche dem Herausragenden keinen Abbruch tun, dürfte ein Zitat aus Kasprowicz' vorletztem Gedicht an den Vierwaldstätter See einen geeigneten Schlusspunkt bilden. Am Ende des Gedichts wird in einem Anruf an „die Wasser“, deren Fels- und Eisumgebung zum Heim des ,großen Geists der Freiheit“ (,wielki duch swobody“) erklärt:

Żegnajcie, wody! [...] duch mój precz ulata

Tam! gdzie się niebo ze szczytami brata,

[...]

Gdzie szumem kaskad groźny twórca świata.

Dech prabytowy, w wiecznych śniegach broczy [...].

Lebt wohl, Ihr Wasser! [...] mein Geist fliegt weiter fort

Dorthin, wo der Himmel sich mit den Gipfeln vereint,

$[\ldots]$

Wo mit Rauschen der strenge Schöpfer der Welt als Wasserfall, der Ur-Atem in ewigen Schnee taucht.

\section{Literatur}

\section{Primärliteratur}

Asnyk, Adam (1880-1894): Poezye; przez El...y. 4 Bd., Hg.: Lwów et al.: Gubrynowicz / Schmidt. Goszczyński, Seweryn (2005): Dziennik podróży do Tatrów. Hg.: Sierotwiński, Stanisław. Wrocław: Zakład Narodowy im. Ossolińskich.

Kasprowicz, Jan (1973): Pisma zebrane. T. 1. Utwory literackie 1. Poezje 1888. Wiersze rozproszone 1877-1888, Hg.: Lipski, Jan Józef. Kraków: Wydawnictwo Literackie.

Konopnicka, Maria (1974): Wrażenia z podróży. In: dies.: Nowele. T. 1, Hg.: Brodzka, Alina. Warszawa: Czytelnik.

Konopnicka, Maria (1926): Sonety wtoskie. In: dies.: Poezye, wydanie zupetne, krytyczne. Opracował Jan Czubek, t. 4: Italia, Warszawa et al.

Prus, Bolesław (1950): Kartki z podróży. 2 Bd., Pisma, t. 27-28, Hg.: Szweykowski, Zygmunt. Warszawa: Książka i Wiedza.

Słowacki, Juliusz (2009): W Szwajcarii. In: ders.: Poematy, 2 Bd., Hg.: Brzozowski, Jacek / Przychodniak, Zbigniew. Poznań: Wydawnictwo Naukowe UAM.

${ }^{24}$ Vgl. zu seiner Lyrik allgemein beispielsweise Kaczyński (1996) oder Loth (1999), zur Eindrücklichkeit der Sprachbilder vgl. Mikołajczyk 2013. 


\section{Sekundärliteratur}

Bischof Hayoz, Judith (2016): Juliusz Stowackis Verserzählungen zwischen Band I Poezye (1832) und den Florentiner Poemen (1838/39): Komposition und Zyklisierungstendenzen. Bern et al.: Lang.

Brang, Peter (1998): Landschaft und Lyrik: die Schweiz in Gedichten der Slaven; eine kommentierte Anthologie. Basel: Schwabe.

Dutka, Elżbieta (2017): „Antologie jako przestrzeń dyskusji na temat literatury „górskiej”. In: Antologia literacka. Przemiany, ekspansja i perspektywy gatunku. Seria pierwsza, S. 213-228.

Igliński, Grzegorz (2008): Fascynacje, inspiracje, koncepcje: romantyczne, pozytywistyczne i młodopolskie obszary wartości i znaczeń. Warszawa: Semper.

Jazowska-Gumulska, Maria (2002): Góry w twórczości Marii Konopnickiej - rozszerzanie przestrzeni. In: Budrewicz, Tadeusz / Zięba, Michał (Hg.): Miejsca Konopnickiej: przeżycia, pejzaż, pamięć. Kraków: Wydaw. Naukowe Akademii Pedagogicznej, 2002, S. 27-37, auch online verfügbar: http://rep.up.krakow.pl/xmlui/bitstream/handle/11716/1733/03--Gory-wtworczosci-Marii-Konopnickiej--Jazowska-Gumulska.pdf?sequence=1\&isAllowed=y (letzter Zugriff: 30.05.2019).

Jochberger, Wolfgang (2004): Ortler — „,der höchste Spiz im ganzen Tirol “. Bozen.

Kaczyński, Jan (Hg.) (1999): Jan Kasprowicz: w siedemdziesięciolecie śmierci. Materiały Międzynarodowej Sesji Naukowej, Olsztyn, 17-19 X 1996, Olsztyn: Wydawnictwo WSP.

Kolbuszewski, Jacek (1971): Obraz Tatr w literaturze polskiej XIX wieku. Funkcja artystyczna motywu przyrody. Kraków: Wydawnictwo Literackie.

Kolbuszewski, Jacek (red.) (1992): Tatry i górale w literaturze polskiej: antologia. Wrocław et al.: Zakład Narodowy im. Ossolińskich.

Kolbuszewski, Jacek (1993): Einladung zu einer Schweizerreise. Das Alpenbild in der polnischen Romantik. In: Nayhauss, Hans-Christoph von (Hg.): Im Dialog mit der interkulturellen Germanistik [Festschrift für Norbert Honsza zum 60. Geburtstag]. Wrocław: Wydawnictwo Uniwersytetu Wrocławskiego, S. 111-122.

Loth, Roman (1994): Jan Kasprowicz. Wrocław et al.: Zakład Narodowy im. Ossolińskich.

Maciejewski, Jarosław (1974): Florenckie poematy Słowackiego. Wrocław et al.: Zakład Narodowy im. Ossolińskich.

Makowski, Stanisław (1976): W szwajcarskich górach. Alpejskie krajobrazy Słowackiego. Warszawa: Państwowy Instytut Wydawniczy.

Markiewicz, Henryk (2006): Pozytywizm 7. Aufl., Warszawa: PWN.

Mazan, Bogdan (2011): , Idee wieczne” i realia kształcace. Przybliżenia z poezji Marii Konopnickiej. In: Wiek XIX. Rocznik Towarzystwa Literackiego im. Adama Mickiewicza XLVI, S. 121-143.

Mikołajczyk, Iwona (2013): Dialektyka obrazowania modernistycznego: „Krzak dzikiej róży w ciemnych smreczynach” J. Kasprowicza i ,„Rozdziobia nas kruki, wrony...." S. Żeromskiego. In: Pamiętnik Literacki 2, S. 137-147.

Mocarska-Tycowa, Zofia (1990): Wybory i konieczności: poezja Asnyka wobec gustów estetycznych i najważniejszych pytań swoich czasów. Toruń: Rozprawy, Uniwersytet Mikołaja Kopernika.

Pawlikowski, Jan Gwalbert (1934): „ZZ dziejów poezji tatrzańskiej”. In: Wierchy XII.

Ritz, German (2012): Nowy polski tekst o Szwajcarii z perspektywy kobiet. Między romantyzmem a modernizmem. In: Wiek XIX. Rocznik Towarzystwa Literackiego im. Adama Mickiewicza XLVII, S. 33-67.

Sawrymowicz, Eugeniusz / Makowski, Stanisław (1960): Kalendarz życia i twórczości Juliusza Słowackiego. Wrocław et al.: Zakład Narodowy im. Ossolińskich.

Sierotwiński, Stanisław (2005): „,Wstęp”. In: Goszczyński, Seweryn: Dziennik podróży do Tatrów. Wrocław: Zakład Narodowy im. Ossolińskich, V-XCVIII. 
Sokólska, Urszula (2008): „,Kilka uwag o konceptualizacji abstraktów w poezji Adama Asnyka”. In: Poradnik Językowy 4, S. 36-49.

Sosnowska, Danuta (2000): Seweryn Goszczyński: biografia duchowa. Wrocław: Fundacja na Rzecz Nauki Polskiej.

Wojtaś, Iwona (2017): Gwiazdy w „Lalce” Bolesława Prusa. In: Pamiętnik Literacki 2, S. 55-71.

Zięba, Michał (1987): „O wydaniach poezji Marii Konopnickiej (szkic informacyjny)”. In: Maria Konopnicka w siedemdziesięciopięciolecie zgonu. Kraków: Wydawnictwo Naukowe Wyższej Szkoły Pedagogicznej, S. 129-146. 